\title{
Dodici nocciole
}

Giovanni Lodi

Dipartimento di Scienze Biomediche, Chirurgiche e Odontoiatriche

Università degli Studi di Milano

via Beldiletto 1/3 Milano 20142

Italia

giovanni.lodi@unimi.it

tel. + 390250319021

fax. +390250319041

Una signora con diversi problemi a denti e gengive mi dice, in perfetta buona fede, che le malattie che l'affliggono sono causate da queste nuove farine che mettono nel pane, "che chissà dove le producono".

Non la sfiora l'idea che invece abbiano a che fare con lo scarso utilizzo dello spazzolino dai tempi in cui le farine le comprava dal mugnaio. L'alimentazione e i suoi effetti sulla salute sono da qualche anno molto pop: un ottimo argomento di conversazione su cui ognuno ha qualche cosa da dire e idee molto chiare, grazie a informazioni raccolte al banco della frutta, sui social o nelle pagine dei quotidiani.

Non di rado, però, le fonti sono autorevoli ricerche, pubblicate da autorevoli riviste, in cui autorevoli scienziati affermano che mangiare dodici nocciole al giorno prolungherebbe la vita di dodici anni, mentre un mandarino al giorno ce ne regalerebbe appena cinque o che, al contrario, un uovo al giorno anticiperebbe la nostra morte di cinque anni e due fette di bacon addirittura di dieci.

Asserzioni raccolte da J.P.A. Ioannidis in un recente editoriale di JAMA sulla ricerca nutrizionale, di cui denuncia i metodi tutt'altro che rigorosi e le semplificazioni eccessive.

Certo, è innegabile che siamo quello che mangiamo e che l'alimentazione ha effetti fondamentali sulla salute: I'obesità ad esempio fa male quasi quanto il fumo. Però da qui a stabilire gli effetti di un particolare alimento sulla lunghezza della nostra vita, o a trasformare i fattori di rischio di una malattia nelle sue cause, ce ne passa.

Perché, come dice loannidis (uno che merita sempre attenzione), pretendere di dimostrare grazie a un questionario gli effetti di una nocciola sugli ottant'anni di vita di un individuo che quotidianamente introduce nell'organismo migliaia di molecole biologicamente attive combinate in milioni di modi, semplicemente non ha senso. E probabilmente produce risultati sbagliati e fuorvianti sulle scelte della gente comune e di chi compila linee guida.

Gli odontoiatri sono stati tra i primi specialisti a fare raccomandazioni sulla dieta, perché da più di un secolo sappiamo che, semplificando appena un po', zucchero più batteri, uguale carie. Ma questa non è la norma, perché nella maggior parte dei casi la relazione tra alimento e malattia è molto meno diretta.

La nostra salute non sarà determinata da una nocciola (con l'eccezione del mio amico Sem che ha uno shock anafilattico solo a sentirne l'odore).

Buona lettura 